\title{
A Note from the Editor-in-Chief
}

Between the time I gave my annual report on the Canadian Journal on Aging (CJA) in October 2007 at the Annual General Meeting of the Canadian Association on Gerontology (CAG) and the end of 2007, what looked like another exceptional year for the $C J A$ turned into a series of production problems with $C J A$ 26(3) 2007, CJA 26(4) 2007, and the CJA Supplement 1 , 2007. The result was that all arrived in 2008. I want to apologize to the authors, our readers, and subscribers. This is unacceptable for all of you and for me as your editor-in-chief.

I also want to express a special apology to Dr. Ed Ansello of Virginia Commonwealth University, one of the guest editors of the CJA Supplement 1, 2007, Hidden Costs/ Invisible Contributions of Caregiving (HCIC), who all had agreed would be listed first in the Table of Contents and in the editorial credits. In fact, the proofs I checked were quite different from what ultimately appeared in the Table of Contents. So, in addition, I would like to apologize to Dr. Janet Fast, the principal investigator of the HCIC team.
This issue of the CJA begins with a guest editorial by Janet Fast, Norah Keating, and Jacquie Eales. A version of this editorial was originally planned as the end piece of the CJA Supplement 1, 2007, Hidden Costs / Invisible Contributions of Caregiving (HCIC). For a number of reasons, I have chosen to publish the editorial as the first piece in this issue to remind readers of the importance of the topic that the HCIC team is tackling and to recommend that even those who read through the supplement when it first came out, go back, and read through it again. There is no doubt in my mind that, no matter how successful formal home care systems are in Canada in the future, informal caregivers will still be critical to the quality of life of seniors as Canada's population ages.

As always, please contact me at mark.rosenberg @queensu.ca if you have any thoughts about this editorial or any other aspect of the CJA.

Mark Rosenberg, Editor-in-Chief 